\title{
1 Rethinking how volatiles are released from plant cells
}

3 Joshua R. Widhalm ${ }^{1}$, Rohit Jaini ${ }^{2}$, John A. Morgan ${ }^{1,2}$, and Natalia Dudareva ${ }^{1,3^{*}}$

4

5 'Department of Biochemistry, Purdue University, 175 South University St., West

6 Lafayette, IN 47907-2063, U.S.A.

$7 \quad{ }^{2}$ School of Chemical Engineering, Purdue University, 480 Stadium Mall Dr., West

8 Lafayette, IN 47907-2100, U.S.A.

$9 \quad{ }^{3}$ Department of Horticulture and Landscape Architecture, Purdue University, 625

10 Agriculture Mall Dr., West Lafayette, IN 47907-2010, U.S.A.

11

12 "Corresponding author: Natalia Dudareva, tel: +1 765494 1325; dudareva@purdue.edu

13

14 Keywords: volatile organic compound (VOC), emission, diffusion,

15 phenylpropanoids/benzenoids, terpenoids, trafficking 


\section{Abstract}

27 For plant volatile organic compounds (VOCs) to be emitted, they must cross

28 membrane(s), the aqueous cell wall, and sometimes the cuticle, before moving into the

29 gas phase. It is presumed that VOC movement through each barrier occurs via passive

30 diffusion. However, VOCs, which are primarily nonpolar compounds, will preferentially

31 partition into membranes, making diffusion into aqueous compartments slow. Using

32 Fick's first law, we calculated that to achieve observed VOC emission rates by diffusion

33 alone would necessitate toxic VOC levels in membranes. We propose that biological

34 mechanisms, like those involved in trafficking other hydrophobic compounds, must

35 contribute to VOC emission. Such parallel biological pathways would lower barrier

36 resistances, thus steady-state emission rates could be maintained with significantly

37 reduced intramembrane VOC concentrations.

38

39

40

41

42

43

44

45

46

47

48

49

50

51

52 


\section{VOCs must cross multiple cellular compartments to reach the environment}

54 Plants emit up to $10 \%$ of photosynthetically fixed carbon in the form of volatile organic

55 compounds (VOCs) [1]. These metabolites, when released from leaves, flowers, and

56 fruits into the atmosphere, and from roots into the soil, play key roles in the attraction of

57 pollinators [2] and seed dispersers [3,4], above- and below-ground defense against

58 herbivores [5-8], protection against pathogens [9,10], and plant-plant signaling [11,12].

59 Certain VOCs are also able to protect plants against abiotic stresses such as high light,

60 temperature, or oxidative stress [13-15]. While VOC functions have long been studied,

61 the last decade has also resulted in substantial progress in the understanding of VOC

62 biosynthesis and regulation [16]. It has been shown that VOC production and release are

63 spatially, developmentally and/or temporally regulated and depend on biotic (e.g.

64 pollination status and herbivore infestation) and abiotic (e.g. light intensity, atmospheric

$65 \mathrm{CO}_{2}$ concentration, temperature, relative humidity, and nutrient status) factors $[13,16,17]$.

66 Based on their biosynthetic origin, plant VOCs can be divided into several

67 classes, including terpenoids (monoterpenes and sesquiterpenes),

68 phenylpropanoids/benzenoids, fatty acid derivatives and amino acid derivatives

$69[13,16,18]$. Despite such chemodiversity, plant VOCs are generally lipophilic low-

70 molecular-weight $(\sim 100-200 \mathrm{Da})$ compounds with high vapor pressures at ambient

71 temperature. These physicochemical properties allow VOCs to be emitted into the

72 environment, although the site from which this occurs varies. The biosynthesis of VOCs

73 in non-green tissues (e.g. flowers or roots) occurs predominantly in epidermal cells,

74 which are in closest proximity to the atmosphere [19-22] or rhizosphere [23] for

75 immediate release. In vegetative organs, VOCs are often synthesized in the secretory

76 cells of glandular trichomes located on the leaf surface [24-27], and then secreted to a

77 sac created by an extension of the cuticle where they are stored until mechanical

78 disruption [28-30]. When trichomes are not involved in vegetative VOC production, these 
79 compounds are often synthesized in mesophyll cells [31,32], mainly in the palisade 80 parenchyma [33], and released through stomata [34-37], mechanical disruption, or 81 emission through cuticle [36]. Regardless of the tissue and if they exit via stomata, at the

82 subcellular level, VOCs must move from their site of biosynthesis through the cytosol to 83 the plasma membrane, and then subsequently traverse the plasma membrane, 84 hydrophilic cell wall, and in some cases the cuticle in order to exit the cell. While 85 mechanical disruption provides direct access for VOCs inside the cell to the atmosphere,

86 it still remains enigmatic how VOCs cross these barriers for release from intact cells

87 directly to the environment or to the intercellular air spaces connected to stomata.

88 It is largely presumed that upon synthesis VOCs passively diffuse across cellular

89 barriers into the environment. Many examples however exist where VOC emission rates

90 cannot be explained by a concentration-dependent diffusion mechanism [38-41].

91 Assuming VOC emission is solely driven by diffusion, we have calculated the

92 concentrations of VOCs at each cellular barrier interface needed to attain published

93 emission rates. Based on the extremely high concentrations of VOCs predicted to

94 accumulate in membranes, we deduce that this would be detrimental to membrane

95 integrity and function. We thus propose that active biological mechanisms are

96 additionally required to lower VOC concentrations in membranes to achieve the

97 observed emission rates. Such mechanisms become even more important under

98 stresses like herbivore attack where VOC emission is drastically increased [42] and

99 membrane integrity is compromised systemically throughout the plant [43]. Finally,

100 based on the cellular trafficking of other hydrophobic compounds (i.e. waxes and 101 diterpenes) in plants we discuss possible biologically controlled routes for VOC export 102 out of cells.

103

104 Diffusion-based models for VOC emission 
105 Over the past couple of decades, VOC emission has been modeled as a diffusion 106 process based on Fick's first law, which states that the steady state flux of any molecule 107 is dictated by the concentration gradient and the resistance of the diffusion path (Box 1).

108 These models have primarily been formulated at the tissue level [44-48], taking into 109 consideration the effects of biosynthesis and environmental factors [49-53]. However,

110 very few studies have looked at VOC movement on the microscopic scale. In their work

111 to investigate the effect of stomatal opening on VOC emission, Niinemets and Reichstein

112 developed a meticulously detailed model taking into account the resistance of individual

113 cellular barriers [51,52]. Barrier resistances were evaluated based on the

114 physicochemical properties of VOCs and structural parameters of each layer. The

115 calculated resistances were then combined to simulate VOC emission and the total 116 internal VOC pools inside the cell.

117 One concern with diffusion-based models, which has not yet been addressed, is

118 that VOCs may accumulate to cytotoxic levels in membranes. Due to their high octanol-

119 water partition coefficients (Box 1, $\mathrm{K}_{\mathrm{o} / \mathrm{w}}$ ), VOCs favorably partition into hydrophobic

120 environments. For small VOCs like isoprene, the diffusivity may be high enough that

121 build up of high concentrations does not occur in membranes. However, for most VOCs

122 to passively diffuse at a physiologically relevant rate into subsequent hydrophilic layers,

123 like the cell wall, they would accumulate to very high levels in membranes. To get an

124 idea if such levels would be biologically tolerable, we calculated the barrier

125 concentrations required to match experimentally measured VOC emission fluxes from

126 snapdragon (Antirrhinum majus) flowers (Box 1) using a similar diffusion-based model

127 as Niinemets and Reichstein [51,52]. Our calculations revealed that the concentration of

128 a given phenylpropanoid/benzenoid or monoterpenoid VOC in the plasma membrane

129 would have to be approximately 50-120 mM to sustain its reported emission flux (Box 1,

130 Table III). This extremely high concentration is primarily due to favorable partitioning of 
131 VOCs into the lipid phase and the high resistance imparted by the cuticle (Box 1, Figure

132 IB). Accumulation of lipophilic molecules in membranes increases permeability, which

133 leads to disruption of proton and ion gradients that are responsible for intracellular $\mathrm{pH}$

134 homeostasis and the driving forces for import and export of compounds out of the cell

135 [54]. Therefore, it is our opinion that biological mechanisms must exist to lower the

136 barrier resistance thereby reducing the VOC concentration in membranes (Box 1). This

137 concept logically extends to interfaces between internal membranes and the cytosol as

138 well. Despite lower VOC emission fluxes, similar mechanisms might also occur in other

139 plant organs, although alternative emission paths must be considered. In leaves, for

140 example, VOCs will readily partition from cell walls into the intercellular gas spaces

141 directly connected to sub-stomatal air spaces, which provide a pathway of lower

142 resistance to diffusion than the cuticle.

144 Possible biological mechanisms involved in VOC emission

145 Based on analogy to intracellular trafficking of other hydrophobic compounds, like waxes

146 and diterpenes, there are several biological mechanisms that could be involved in

147 shuttling VOCs out of undamaged cells [55]. VOCs synthesized in the cytosol will

148 favorably partition into both plasma membranes and subcellular membranes (Figure 1).

149 VOCs in internal membranes could be trafficked to the plasma membrane via ER-

150 plasma membrane contact sites or via vesicle trafficking processes associated with the

151 ER and Golgi, trans-Golgi network (TGN), and/or vacuole (Figure 1). These processes

152 would also effectively reduce the accumulation of VOCs in internal membranes.

153 Although vesicular transport of metabolites is relatively unexplored in plants, it is already

154 thought to play a role in the movement of cytotoxic phytochemicals [56,57], hormones

155 [58], anti-microbial compounds [59,60], pigments [61] and cuticular wax components

$156[62,63]$. VOCs synthesized in other organelles, such as monoterpenes in plastids, could 
157 also be delivered to the ER via interorganellar membrane hemifusion (Figure 1), a

158 mechanism recently proposed for the transfer of lipophilic metabolites between plastids

159 and ER $[64,65]$. Alternatively, VOC trafficking to the plasma membrane could be

160 mediated by soluble carrier proteins with hydrophobic pockets capable of escorting

161 lipophilic compounds across aqueous environments [66] (Figure 1).

162 The subsequent translocation of VOCs across the plasma membrane to the

163 apoplast entails the movement of hydrophobic molecules across a lipophilic layer into an

164 aqueous compartment. One possibility is that plasma membrane localized transporters

165 are involved in directional export of VOCs out of the cell. Recently, it has been proposed

166 that vesicles carrying cuticle wax components deliver their cargo to plasma membrane

167 ATP binding cassette (ABC) transporters for export to the cell exterior [63,67]. Moreover,

168 an Arabidopsis thaliana plasma membrane ABC transporter, AtABCG29, has been

169 shown to transport the monolignol $p$-coumaryl alcohol [68], which is structurally similar

170 and shares the same core biosynthetic pathway as the VOCs isoeugenol, eugenol, and

171 vanillin emitted from Petunia hybrida flowers [69]. Remarkably, expression of PhABCG1,

172 a petunia AtABCG29 homolog encoding a plasma membrane localized transporter with

173 unknown function, is regulated by the ODORANT1 (ODO1) transcription factor [70].

174 Because the ODO1 transcription factor is known to control several biosynthetic genes

175 encoding enzymes involved in synthesizing precursors for phenylpropanoid/benzenoid

176 VOCs, it will be important to determine whether PhABCG1 exports VOCs to the cell wall.

177 Once VOCs cross the lipophilic plasma membrane, they must traverse the hydrophilic

178 cell wall. Although our diffusion model (Box 1 ) does not indicate the cell wall exerts a

179 large resistance per se, small carrier proteins, such as extracellular lipid transfer proteins

180 (LTPs), may be necessary to prevent repartitioning of VOCs into the plasma membrane

181 (Figure 1). In plants, LTPs have recently been shown to function in the export of wax

182 lipids and diterpenes from the plasma membrane, across the cell wall, to the cuticle [71- 
183 74]. Since LTPs generally lack substrate specificity, it is possible that the same LTPs 184 may also be transporting VOCs to neighboring cells, intercellular spaces (in some cases

185 for release via stomata), or the cuticle. The concentration of VOCs in the cuticle and

186 their rate of release into the environment are dependent on the cuticle composition and

187 its thickness [40, 75-77]. This is consistent with our calculations showing that the cuticle

188 imposes the largest resistance to VOC emission (Box 1, Figure IB). In the presence of

189 biological mechanisms at the other cellular barriers, the control of the cuticle on emission

190 will be even higher.

191

\section{Concluding Remarks}

193 In order for VOCs to be released from intact cells into the environment they must cross

194 several subcellular barriers, each providing resistance to the overall emission rate.

195 Examination of existing diffusion models strongly suggests that to drive observed

196 emission rates high VOC levels would need to accumulate in the hydrophobic

197 compartments that these molecules must traverse. Although we cannot exclude the

198 possibility that VOC emission occurs via a solely diffusion-dependent process, it appears

199 unlikely that this would be the case given the detrimental effects of high VOC 200 concentrations in membranes. Therefore, it seems more plausible that there are

201 biological mechanisms in place to facilitate emission flux with reduced VOC membrane

202 levels. In the presence of biological control, the movement of VOCs through different 203 barriers is analogous to current flow through circuits with parallel resistances, $r_{\text {biological }}$ or $204 r_{\text {diffusive, }}$ in which the current will flow preferentially through the path of least resistance. In 205 light of recent progress made in both deep sequencing technologies and understanding 206 of intracellular transport of other hydrophobic compounds, we propose future research 207 should explore if similar mechanisms are involved in subcellular VOC trafficking. Since 208 the cuticle imposes the largest resistance to VOC emission, another important area of 
209 research that should be further investigated using genetic approaches is the impact of 210 cuticle composition on VOC emission.

211

212 Acknowledgements

213 We thank Dr. Sergei Savikhin for checking diffusion model simulations. This work was

214 supported by grant DE-SC0008628 from the U.S. Department of Energy to N.D. and

215 J.A.M., and by the Gordon and Betty Moore Foundation through grant GBMF2550.02 to

216 the Life Sciences Research Foundation to J.R.W.

217

218 Disclaimer statement

219 The authors declare there is no conflict of interest.

220

221

222

223

224

225

226

227

228

229

230

231

232 


\section{References}

234 1. Penuelas, J. and Llusia, J. (2004) Plant VOC emissions: making use of the 235 unavoidable. Trends Ecol. and Evol., 19, 402-404

236 2. Raguso, R. (2008) Wake up and smell the roses: The ecology and evolution of floral 237 scent. Annu. Rev. Ecol. Evol. Syst. 39, 549-569

238 3. Bolen, R. and Green, S. (1997) Use of olfactory cues in foraging by owl monkeys 239 (Aotus nancymai) and capuchin monkeys (Cebus apella). J. Comp. Psychol. 111, $240 \quad 152-158$

241 4. Luft, S. et al. (2003) The use of olfaction in the foraging behaviour of the golden242 mantled flying fox, Pteropus pumilus, and the greater musky fruit bat, Ptenochirus

243 jagori (Megachiroptera : Pteropodidae). Naturwissenschaften 90, 84-87

244 5. Degenhardt, J. et al. (2009) Restoring a maize root signal that attracts insect-killing 245 nematodes to control a major pest. Proc. Natl. Acad. Sci. U.S.A. 106, 17606-17606

246 6. Hiltpold, I. and Turlings, T. (2012) Manipulation of chemically mediated interactions

247 in agricultural soils to enhance the control of crop pests and to improve crop yield. J.

$248 \quad$ Chem. Ecol. 38, 641-650

249 7. Kappers, I. et al. (2011) Variation in herbivory-induced volatiles among cucumber 250 (Cucumis sativus L.) varieties has consequences for the attraction of carnivorous 251 natural enemies. J. Chem. Ecol. 37, 150-160

252 8. Unsicker, S. et al. (2009) Protective perfumes: the role of vegetative volatiles in 253 plant defense against herbivores. Curr. Opin. Plant Biol. 12, 479-485

254 9. Huang, M. et al. (2012) The major volatile organic compound emitted from

255 Arabidopsis thaliana flowers, the sesquiterpene (E)-beta-caryophyllene, is a defense 256 against a bacterial pathogen. New Phytol. 193, 997-1008 
257 10. Keeling, C. and Bohlmann, J. (2006) Genes, enzymes and chemicals of terpenoid 258 diversity in the constitutive and induced defense of conifers against insects and 259 pathogens. New Phytol. 170, 657-675

260 11. Baldwin, I. et al. (2006) Volatile signaling in plant-plant interactions: "Talking trees" in 261 the genomics era. Science $311,812-815$

262 12. Heil, M. and Bueno, J.C.S. (2007) Within-plant signaling by volatiles leads to

263 induction and priming of an indirect plant defense in nature. Proc. Natl. Acad. Sci.

264 U.S.A. $104,5467-5472$

265 13. Dudareva, N. et al. (2006) Plant volatiles: Recent advances and future perspectives.

266 Crit. Rev. Plant Sci. 25, 417-440

267 14. Holopainen, J. (2011) Can forest trees compensate for stress-generated growth

268 losses by induced production of volatile compounds? Tree Physiol. 31, 1356-1377

269 15. Vickers, C. et al. (2009) A unified mechanism of action for volatile isoprenoids in

270 plant abiotic stress. Nature Chem. Biol. 5, 283-291

271 16. Dudareva, N. et al. (2013) Biosynthesis, function and metabolic engineering of plant

272 volatile organic compounds. New Phytol. 198, 16-32

273 17. Holopainen, J. and Gershenzon, J. (2010) Multiple stress factors and the emission of 274 plant VOCs. Trends Plant Sci. 15, 176-184

275 18. Knudsen, J. et al. (2006) Diversity and distribution of floral scent. Bot. Rev. 72, 1-120

276 19. Dudareva, N. et al. (1996) Evolution of floral scent in Clarkia: Novel patterns of S-

277 linalool synthase gene expression in the C. breweri flower. Plant Cell 8, 1137-1148

278 20. Effmert, U. et al. (2006) Localization of the synthesis and emission of scent

279 compounds within the flower. In Biology of Floral Scent (Dudareva, N. and Pichersky, $280 \quad$ E., eds), pp. 105-124, CRC Press

281 21. Kolosova, N. et al. (2001) Cellular and subcellular localization of S-adenosyl-L-

282 methionine: benzoic acid carboxyl methyltransferase, the enzyme responsible for 
283 biosynthesis of the volatile ester methylbenzoate in snapdragon flowers. Plant 284 Physiol. 126, 956-964

285 22. Scalliet, G. et al. (2006) Role of petal-specific orcinol O-methyltransferases in the 286 evolution of rose scent. Plant Physiol. 140, 18-29

287 23. Chen, F. et al. (2004) Characterization of a root-specific Arabidopsis terpene

288 synthase responsible for the formation of the volatile monoterpene 1,8-cineole. Plant

289 Physiol. 135, 1956-1966

290 24. Lu, S. et al. (2002) Cloning and functional characterization of a beta-pinene synthase

291 from Artemisia annua that shows a circadian pattern of expression. Plant Physiol.

$292 \quad 130,477-486$

293 25. McCaskill, D. et al. (1992) Morphology and monoterpene biosynthetic capabilities of

294 secretory-cell clusters isolated from glandular trichomes of peppermint (Mentha

295 piperita I). Planta $187,445-454$

296 26. Schilmiller, A. et al. (2009) Monoterpenes in the glandular trichomes of tomato are

297 synthesized from a neryl diphosphate precursor rather than geranyl diphosphate.

298 Proc. Natl. Acad. Sci. U.S.A. 106, 10865-10870

299 27. Wang, G. et al. (2008) Terpene biosynthesis in glandular trichomes of hop. Plant

$300 \quad$ Physiol. 148, 1254-1266

301 28. Gang, D. et al. (2001) An investigation of the storage and biosynthesis of

302 phenylpropenes in sweet basil. Plant Physiol. 125, 539-555

303 29. lijima, Y. et al. (2004) The biochemical and molecular basis for the divergent patterns

304 in the biosynthesis of terpenes and phenylpropenes in the peltate glands of three

305 cultivars of basil. Plant Physiol. 136, 3724-3736

306 30. Turner, G. et al. (2000) Distribution of peltate glandular trichomes on developing

307 leaves of peppermint. Plant Physiol. 124, 655-663 
308 31. Koeduka, T. et al. (2009) Biosynthesis of $t$-anethole in Anise: Characterization of $t$ -

309 anol/isoeugenol synthase and an O-methyltransferase specific for a C7-C8 propenyl

$310 \quad$ side chain. Plant Physiol. 149, 384-394

311 32. Kollner, T. et al. (2013) Localization of sesquiterpene formation and emission in

312 maize leaves after herbivore damage. BMC Plant Biol. 13, 15

313 33. Cinege, G. et al. (2009) Regulation of isoprene synthase promoter by environmental

314 and internal factors. Plant Mol. Biol. 69, 593-604

315 34. Kesselmeier, J. and Staudt, M. (1999) Biogenic volatile organic compounds (VOC):

316 An overview on emission, physiology and ecology. J. Atmos. Chem. 33, 23-88

317 35. Niinemets, U. et al. (2004) Physiological and physicochemical controls on foliar

318 volatile organic compound emissions. Trends Plant Sci. 9, 180-186

319 36. Niinemets, U. et al. (2002) Stomatal constraints may affect emission of oxygenated

320 monoterpenoids from the foliage of Pinus pinea. Plant Physiol. 130, 1371-1385

321 37. Seidl-Adams, I. et al. (2014) Emission of herbivore elicitor-induced sesquiterpenes is

322 regulated by stomatal aperture in maize (Zea mays) seedlings. Plant Cell Environ.

$323 \quad 38,23-34$

324 38. Ben Zvi, M. et al. (2008) Interlinking showy traits: co-engineering of scent and colour

325 biosynthesis in flowers. Plant Biotechnol. J. 6, 403-415

326 39. Gershenzon, J. et al. (2000) Regulation of monoterpene accumulation in leaves of

327 peppermint. Plant Physiol. 122, 205-213

328 40. Goodwin, S. et al. (2003) Cuticle characteristics and volatile emissions of petals in

$329 \quad$ Antirrhinum majus. Physiol. Plantarum 117, 435-443

330 41. Sagae, M. et al. (2008) Effect of temperature on the floral scent emission and

331 endogenous volatile profile of Petunia axillaris. Biosci. Biotechnol. Biochem. 72, 110-

$332 \quad 115$ 
333 42. Hare, J.D. (2011) Ecological role of volatiles produced by plants in response to 334 damage by herbivorous insects. Annu. Rev. Entomol. 56, 161-180

335 43. Walker-Simmons, M. et al. (1984) Wound signals in plants: A systemic plant would 336 signal alters plasma membrane intergrity. Proc. Natl. Acad. Sci. USA 81, 3737-3741

337 44. Guenther, A. et al. (1994) Natural volatile organic compound emission rate estimates

338 for U.S. woodland landscapes. Atmos. Environ. 28, 1197-1210

339 45. Bai, J. et al. (2006) Isoprene and monoterpene emissions from an Inner Mongolia

$340 \quad$ grassland. Atmos. Environ. 40, 5753-5758

341 46. Geron, C. et al. (2006) Biogenic volatile organic compound emissions from desert

342 vegetation of the southwestern US. Atmos. Environ. 40, 1645-1660

343 47. Harley, P. et al. (2004) Variation in potential for isoprene emissions among

$344 \quad$ Neotropical forest sites. Glob. Chang. Biol. 10, 630-650

345 48. Kuhn, U. et al. (2004) Seasonal differences in isoprene and light-dependent

346 monoterpene emission by Amazonian tree species. Glob. Chang. Biol. 10, 663-682

347 49. Zimmer, W. et al. (2003) The process-based SIM-BIM model: towards more realistic

348 prediction of isoprene emissions from adult Quercus petraea forest trees. Atmos.

$349 \quad$ Environ. 37, 1665-1671

350 50. Niinemets, Ü. et al. (2010) The emission factor of volatile isoprenoids: stress,

351 acclimation, and developmental responses. Biogeosciences 7, 2203-2223

352 51. Niinemets, Ü. and Reichstein, M. (2003) Controls on the emission of plant volatiles

353 through stomata: Differential sensitivity of emission rates to stomatal closure

354 explained. J. Geophys. Res. 108 (D7), 4208, DOI:10.1029/2002JD002626

355 52. Niinemets, Ü. and Reichstein, M. (2003) Controls on the emission of plant volatiles

356 through stomata: A sensitivity analysis. J. Geophys. Res. 108 (D7), 4211,

357 DOI: 10.1029/2002JD002626 
53. Martin, M.J. et al. (2000) A process-based model to predict the effects of climatic

359 change on leaf isoprene emission rates. Ecol. Modell. 131, 161-174

360 54. Sikkema, J. et al. (1995) Mechanisms of membrane toxicity of hydrocarbons.

361 Microbiol. Mol. Biol. Rev. 59, 201-222

362 55. Jetter, R. (2006) Examination of the processes involved in the emission of scent

363 volatiles from flowers. In Biology of Floral Scent (Dudareva, N. and Pichersky, E.,

364 eds), pp. 125-144, CRC Press

365 56. Facchini, P. (2001) Alkaloid biosynthesis in plants: Biochemistry, cell biology,

366 molecular regulation, and metabolic engineering applications. Ann. Rev. Plant

367 Physiol. Plant Mol. Biol. 52, 29-66

368 57. Weston, L. et al. (2012) Mechanisms for cellular transport and release of

369 allelochemicals from plant roots into the rhizosphere. J. Exp. Bot. 63, 3445-3454

370 58. Geldner, N. et al. (2001) Auxin transport inhibitors block PIN1 cycling and vesicle

371 trafficking. Nature $413,425-428$

372 59. Kwon, C. et al. (2008) Secretory pathways in plant immune responses. Plant Physiol.

$373 \quad 147,1575-1583$

374 60. Robatzek, S. (2007) Vesicle trafficking in plant immune responses. Cell. Microbiol. 9, $375 \quad 1-8$

376 61. Zhao, J. and Dixon, R. (2010) The 'ins' and 'outs' of flavonoid transport. Trends Plant 377 Sci. $15,72-80$

378 62. Samuels L. et al. (2008) Sealing plant surfaces: cuticular wax formation by epidermal 379 cells. Ann. Rev. Plant Biol. 59, 683-707

380 63. McFarlane, H. et al. (2014) Golgi- and trans-golgi network-mediated vesicle

381 trafficking is required for wax secretion from epidermal cells. Plant Physiol. 164,

$382 \quad 1250-1260$ 
383 64. Mehrshahi, P. et al. (2014) Redefining the metabolic continuity of chloroplasts and

$384 \quad$ ER. Trends Plant Sci. 19, 501-507

385 65. Mehrshahi, P. et al. (2013) Transorganellar complementation redefines the

386 biochemical continuity of endoplasmic reticulum and chloroplasts. Proc. Natl. Acad.

$387 \quad$ Sci. U.S.A. $110,12126-12131$

388 66. Samuels, L. and McFarlane, H. (2012) Plant cell wall secretion and lipid traffic at

389 membrane contact sites of the cell cortex. Protoplasma 249, S19-S23

390 67. McFarlane, H. et al. (2010) Arabidopsis ABCG transporters, which are required for

391 export of diverse cuticular lipids, dimerize in different combinations. Plant Cell 22,

$392 \quad 3066-3075$

393 68. Alejandro, S. et al. (2012) AtABCG29 is a monolignol transporter involved in lignin

394 biosynthesis. Curr. Biol. 22, 1207-1212

395 69. Muhlemann, J.K. et al. (2014) The monolignol pathway contributes to the

396 biosynthesis of volatile phenylpropenes in flowers. New Phytol. 204, 661-670

397 70. Van Moerkercke, A. et al. (2012) Regulators of floral fragrance production and their

398 target genes in petunia are not exclusively active in the epidermal cells of petals. $J$.

$399 \quad$ Exp. Bot. 63, 3157-3171

400 71. Cameron, K.D. et al. (2006) Increased accumulation of cuticular wax and expression

401 of lipid transfer protein in response to periodic drying events in leaves of tree

402 tobacco. Plant Physiol. 140, 176-183

403 72. Choi, Y. et al. (2012) Tobacco NtLTP1, a glandular-specific lipid transfer protein, is

404 required for lipid secretion from glandular trichomes. Plant J. 70, 480-491

405 73. DeBono, A. et al. (2009) Arabidopsis LTPG Is a glycosylphosphatidylinositol-

406 anchored lipid transfer protein required for export of lipids to the plant surface. Plant

407 Cell 21, 1230-1238 
74. Lee, S. et al. (2009) Disruption of glycosylphosphatidylinositol-anchored lipid transfer

409 protein gene altered cuticular lipid composition, increased plastoglobules, and

410 enhanced susceptibility to infection by the fungal pathogen Alternaria brassicicola.

$411 \quad$ Plant Physiol. 150, 42-54

412 75. Jeffree, C. E. (2006) The fine structure of the plant cuticle. Ann. Plant Rev., 23, 11 -

$413 \quad 125$

414 76. Leide, J. et al. (2007) The developmental pattern of tomato fruit wax accumulation

415 and its impact on cuticular transpiration barrier properties: Effects of a deficiency in a

416 beta-ketoacyl-coenzyme A synthase (LeCER6). Plant Physiol. 144,1667-1679

417 77. Martin, L.B.B. and Rose, J.K.C. (2014) There's more than one way to skin a fruit:

418 formation and functions of fruit cuticles. J. Exp. Bot. 65, 4639-4651

419 78.Nagegowda, D. et al. (2008) Two nearly identical terpene synthases catalyze the

420 formation of nerolidol and linalool in snapdragon flowers. Plant J. 55, 224-239

421 79. Popp, C. et al. (2005) Characterization of hydrophilic and lipophilic pathways of

422 Hedera helix L. cuticular membranes: permeation of water and uncharged organic

423 compounds. J. Exp. Bot. 56, 2797-806

424 80. Schreiber, L. et al. (1996) Transport properties of cuticular waxes of Fagus sylvatica

425 L . and Picea abies ( L .) Karst .: Estimation of size selectivity and tortuosity from

426 diffusion coefficients of aliphatic molecules. Planta 198, 104-109

427 81. Gharagheizi, F. (2012) Determination of diffusion coefficient of organic compounds in

428 water using a simple molecular-based method. Ind. Eng. Chem. Res. 51, 2797-2803

429 82. Walter, A and Gutknecht, J. (1986) Permeability of small nonelectrolytes through

$430 \quad$ lipid bilayer membranes. J. Membr. Biol. 90, 207-17

431 83. Berthod, A. and Carda-Broch, S. (2004) Determination of liquid-liquid partition

432 coefficients by separation methods. J. Chromatogr. A 1037, 3-14 
433 84. Camargos, H.S. et al. (2014) Terpenes increase the lipid dynamics in the

434 Leishmania plasma membrane at concentrations similar to their $\mathrm{IC}_{50}$ values. PLoS

$435 \quad$ One 9, e104429. doi:10.1371/journal.pone.0104429

436 85. Hine, R. (1999) Membrane. In The facts on file dictionary of biology (3 $3^{\text {rd }}$ edn), p. 198,

$437 \quad$ Checkmark

438

439

440

441 Figure legend

442 Figure 1. Proposed models for VOC trafficking in plant cells. VOCs may diffuse through

443 each cell barrier, or be trafficked via unknown biological mechanisms similar to that of

444 other hydrophobic compounds [55-74]. The physicochemical parameters of most VOCs

445 likely preclude their movement through the cell solely by diffusion. Possible vesicle

446 tracking routes for transport through the cytosol are depicted by gray block arrows. Lipid

447 transfer proteins (LTPs) or other types of carrier proteins may contribute to shuttling

448 VOCs through the plastid stroma, cytosol, and/or cell wall. Transporters localized in the

449 plasma membrane may play a role in exporting VOCs from the cytosol or membrane into

450 the cell wall. VOCs then diffuse through the cuticle for emission into the environment.

451 The involvement of other biological mechanisms should not be excluded. Abbreviations:

452 GPI, glycosylphosphatidylinositol.

454 Box 1. VOC diffusion at the subcellular level according to Fick's first law

455 At the subcellular level, VOCs synthesized in the cytosol must cross the plasma 456 membrane and cell wall barriers, and in some cases the cuticle, to be emitted (Figure 
457 IA). If diffusion is the sole driving force of VOC emission, then under steady-state 458 conditions, emission flux ( $J$ ) can be modeled by Fick's first law as (equation I):

$$
J=\frac{\Delta C}{r_{i}}
$$

460 where $\Delta C$ represents the concentration difference along the diffusion path and $r_{i}$ is the

461 resistance to diffusion through a given barrier, $i$ (Figure I). The resistances through each

462 barrier are a function of length, $l_{i}$, and VOC physicochemical properties, which include

463 diffusivity through a given barrier, $D_{i}$, and octanol-water $\left(K_{o / w}\right)$ and cuticle-water $\left(K_{c / w}\right)$

464 partition coefficients, (ratios of concentrations of a compound in the octanol phase and 465 cuticle, respectively, to its equilibrium aqueous-phase concentration) as follows 466 (equations II).

470 diffusion, we investigated the VOC concentrations in each subcellular barrier required to

471 match experimentally measured VOC emission fluxes from snapdragon flowers $[40,78]$.

472 For illustrative purposes, we modeled diffusion of a representative 473 benzenoid/phenylpropanoid (methylbenzoate) and terpenoid (nerolidol) VOC, both of 474 which are synthesized in cytosol $[21,78]$. Thus, internal membrane barrier resistances 475 (e.g. plastid) were not considered. Since flowers lack stomata, and VOC biosynthesis 476 occurs in epidermal cells, VOC fluxes into intercellular air spaces were not factored.

477 Barrier resistances were calculated using equation II with parameters available from the 478 literature (Tables I and II). Concentrations in each barrier were solved assuming uniform 479 VOC concentrations in the cytosol and neglecting the bulk concentration $\left(\mathrm{C}_{\text {bulk }}\right)$ of VOCs 480 at the air-cuticle interface. Combining these assumptions with steady-state mass 
481 balance at each barrier interface, we solved for VOC concentrations as follows (equation 482 III):

$$
J_{s y n}=\frac{\left(C_{C y t}-C_{C W 1}\right)}{r_{P M}}=\frac{\left(C_{C W 1}-C_{C W 2}\right)}{r_{C W}}=\frac{C_{C u t 1}}{r_{C u t}}=J_{e m i}
$$

485 membrane as a result of its short path length and/or the high diffusivity of VOCs in this

486 layer. Our calculations further indicate that to match measured emission fluxes $[40,78]$,

487 methylbenzoate and nerolidol would need to hyper-accumulate in the plasma membrane

488 (Figure IB, Table III) to levels that are likely detrimental to the cell. Therefore, we 489 propose that the concentration of VOCs in cellular membranes has to be lowered by

490 biological mechanisms (Figure 1). Our simulation also indicates that the resistance

491 imposed by the cuticle is much higher than that of the plasma membrane and cell wall, 492 suggesting that it serves as the rate-limiting barrier to VOC emission.

\section{Box Figure Legend and Tables}

497 Figure I. (A) Diagram depicting VOC emission barriers in snapdragon flowers. (B) 498 Simulated VOC concentrations (equation III) within each barrier needed to match 499 measured emission rates of methylbenzoate (black line) [40] and nerolidol (blue line)

500 [78] solely by passive diffusion. See Table III for calculated concentration values. 
506 Table I: VOC physicochemical properties and fluxes

\begin{tabular}{|c|c|c|c|c|c|}
\hline \multirow{3}{*}{$\begin{array}{l}\text { VOC Property } \\
\text { Diffusivity in cuticle }^{b}\end{array}$} & \multirow{3}{*}{$\begin{array}{l}\text { Symbol (Unit) } \\
D_{\text {Cut }}\left(m^{2} s^{-1}\right)\end{array}$} & \multicolumn{4}{|c|}{ Value $^{\mathrm{a}}$ [Reference] } \\
\hline & & \multicolumn{2}{|c|}{ Methylbenzoate } & \multicolumn{2}{|c|}{ Nerolidol } \\
\hline & & $10^{-16}$ & {$[79]$} & $10^{-17}$ & [80] \\
\hline Diffusivity in cell wall & $D_{C w}\left(m^{2} s^{-1}\right)$ & $7 \times 10^{-10}$ & [81] & $5 \times 10^{-10}$ & [81] \\
\hline Diffusivity in plasma membrane ${ }^{b}$ & $D_{P M}\left(m^{2} s^{-1}\right)$ & $2 \times 10^{-10}$ & [82] & $10^{-7}$ & [82] \\
\hline Octanol-water partition coefficient & $\mathrm{K}_{\mathrm{o} / \mathrm{w}}$ & $10^{2.1}$ & {$[83]$} & $10^{5.3}$ & [84] \\
\hline Cuticle-water partition coefficient $^{b}$ & $\mathrm{~K}_{\mathrm{c} / \mathrm{w}}$ & $10^{1.1}$ & [79] & $10^{4.3}$ & [79] \\
\hline Emission Flux & $J_{e m i}\left(\mathrm{~mol} \mathrm{~m}^{-2} \mathrm{~s}^{-1}\right)$ & $7.5 \times 10^{-9}$ & {$[40]$} & $3 \times 10^{-10}$ & [78] \\
\hline
\end{tabular}

507 aaccurate to within an order of magnitude

508 bdetermined using empirical relations developed in respective references

509

510 Table II: Tissue structural properties

\begin{tabular}{|c|c|c|c|}
\hline Characteristic & Symbol (Unit) & \multicolumn{2}{|c|}{ Value (Reference) } \\
\hline Cuticle diffusion path length & $\mathrm{I}_{\text {Cut }}(\mathrm{m})$ & $1.6 \times 10^{-7}$ & [40] \\
\hline Cell wall diffusion path length & $\mathrm{I}_{c W}(\mathrm{~m})$ & $4 \times 10^{-7}$ & [40] \\
\hline Plasma membrane thickness & $\mathrm{I}_{P M}(\mathrm{~m})$ & $10^{-8}$ & [85] \\
\hline Total petal area & $A_{p}\left(\mathrm{~m}^{2}\right)$ & $7 \times 10^{-4}$ & [40] \\
\hline
\end{tabular}

515 Table III: VOC concentrations in each barrier required to match emission flux

\section{6 solely by passive diffusion}

\begin{tabular}{l|l|l|l|l|}
\multirow{2}{*}{ Barrier } & \multicolumn{3}{l}{ Concentration $(\mathrm{C})$} \\
\cline { 2 - 5 } & \multicolumn{3}{l}{ Methylbenzoate } & \multicolumn{2}{l}{ Nerolidol } \\
\cline { 2 - 5 } & $\mathrm{C}_{1}$ & $\mathrm{C}_{2}$ & $\mathrm{C}_{1}$ & $\mathrm{C}_{2}$ \\
\hline Cytosol & $0.95 \mathrm{mM}$ & $\mathrm{n} / \mathrm{a}$ & $0.24 \mu \mathrm{M}$ & $\mathrm{n} / \mathrm{a}$ \\
\hline Plasma membrane & $120 \mathrm{mM}$ & $120 \mathrm{mM}$ & $48 \mathrm{mM}$ & $48 \mathrm{mM}$ \\
\hline Cell wall & $0.95 \mathrm{mM}$ & $0.95 \mathrm{mM}$ & $0.24 \mu \mathrm{M}$ & $0.24 \mu \mathrm{M}$ \\
\hline Cuticle & $12 \mathrm{mM}$ & 0 & $4.8 \mathrm{mM}$ & 0
\end{tabular}




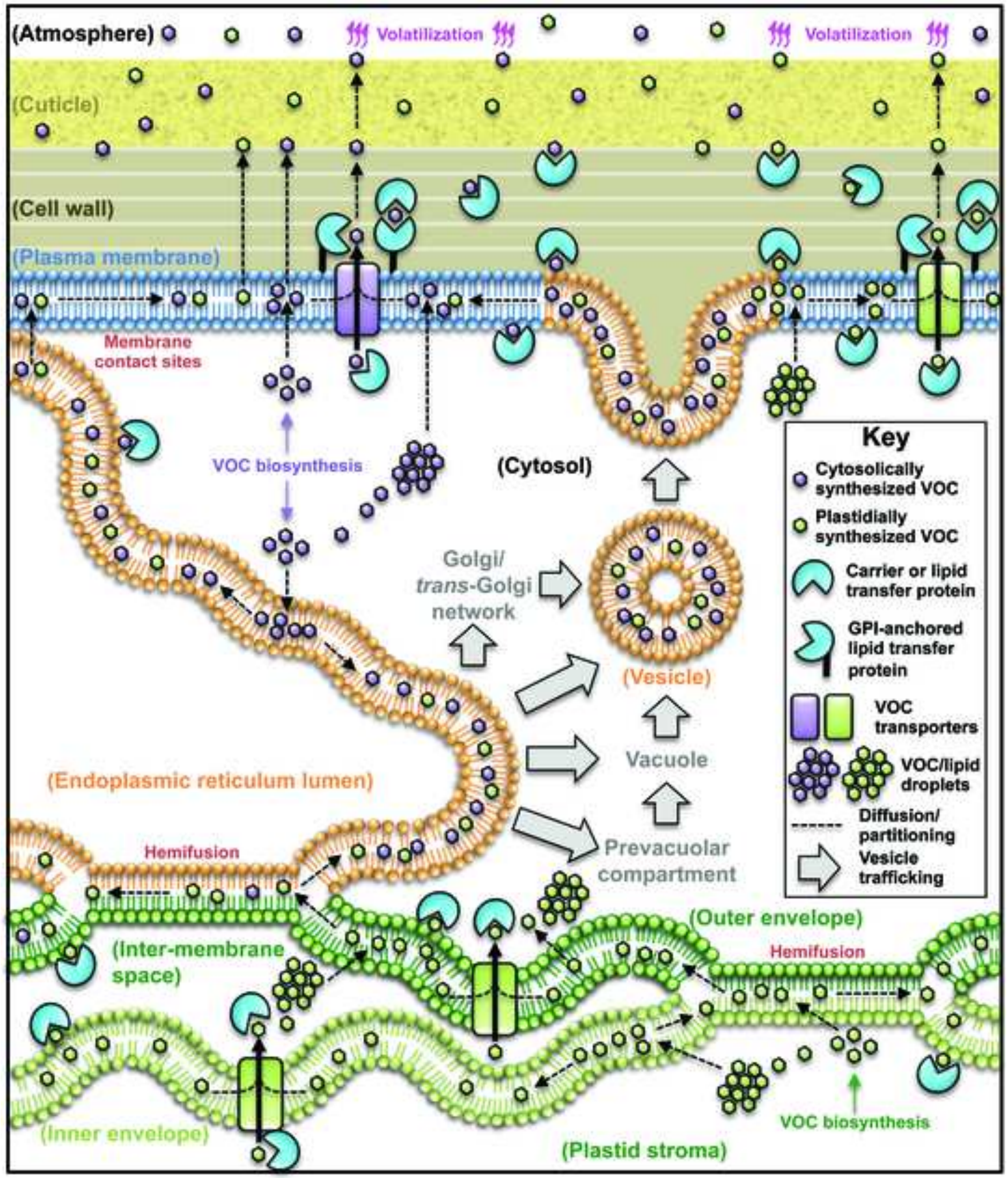


(A)

Plasma

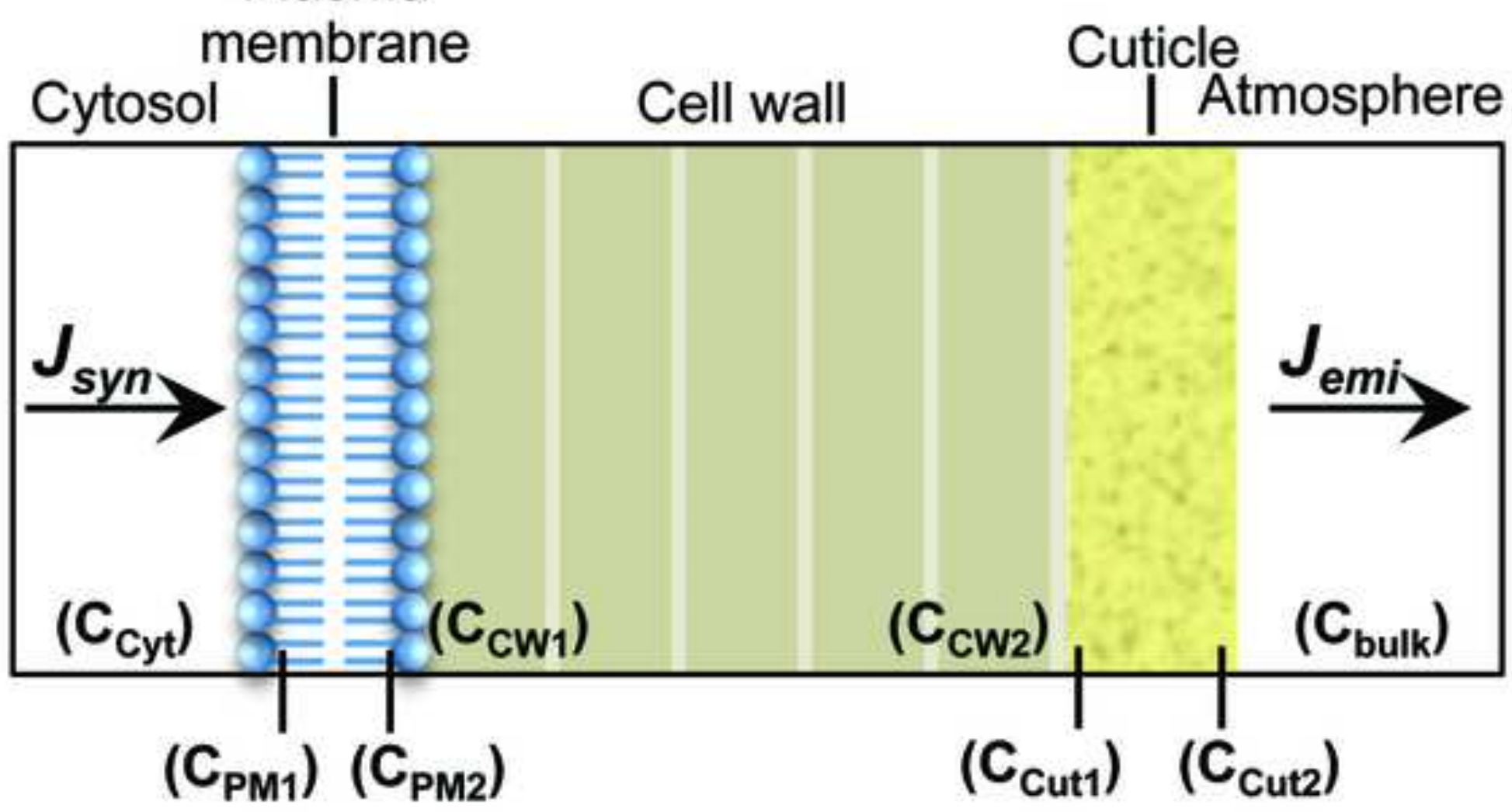

(B)

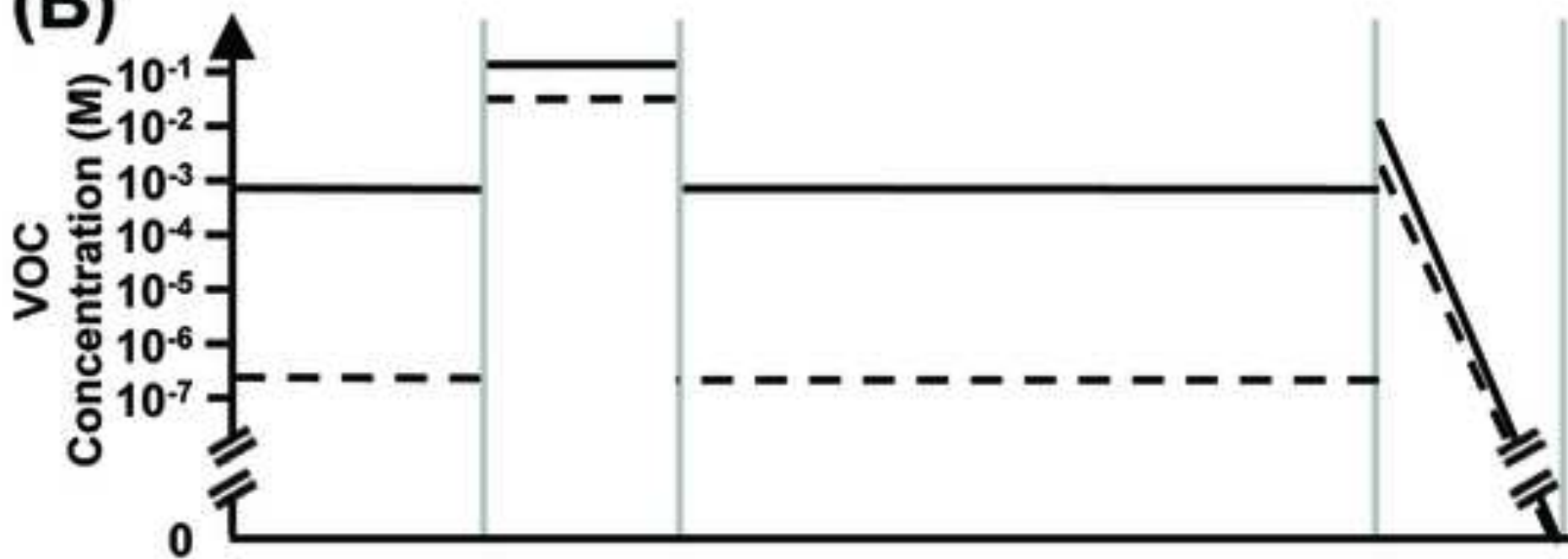

